\title{
A comparison of arterial blood glucose and peripheral blood glucose levels in critically ill patients: measurements using the arterial blood gas analyzer and the rapid glucose meter
}

\author{
Ting Deng ${ }^{1}$, Mengjiao Liu ${ }^{1}$, Lu Pan ${ }^{1}$, Kai Jiang ${ }^{2}, \mathrm{Yi} \mathrm{Li}^{3}$ \\ ${ }^{1}$ Clinical Nursing Teaching and Research Section, The Second Xiangya Hospital, Central South University, Changsha, China; ${ }^{2}$ Department of \\ Orthopedics, The Second Xiangya Hospital, Central South University, Changsha, China; ${ }^{3}$ Department of Critical Care Medicine, The Second \\ Xiangya Hospital, Central South University, Changsha, China \\ Contributions: (I) Conception and design: M Liu, L Pan; (II) Administrative support: T Deng; (III) Provision of study materials or patients: Y Li; (IV) \\ Collection and assembly of data: T Deng, Y Li; (V) Data analysis and interpretation: M Liu, K Jiang; (VI) Manuscript writing: All authors; (VII) \\ Final approval of manuscript: All authors. \\ Correspondence to: Mengjiao Liu. Clinical Nursing Teaching and Research Section, The Second Xiangya Hospital, Central South University, No. 139, \\ Renmin Middle Road, Furong District, Changsha 410011, China. Email: liumengjiao@csu.edu.cn.
}

\begin{abstract}
Background: This study explored the differences, correlation, and consistency between blood glucose levels measured using an arterial blood gas analyzer and a rapid blood glucose meter in critically ill patients.

Methods: A total of 73 critically ill patients admitted to the Department of Critical Care Medicine, from October to December 2016 were enrolled in this study. The patient's arterial blood glucose was measured by arterial blood gas analyzer, while peripheral blood glucose was measured by a rapid blood glucose meter (via the non-infusion limb). The correlation between indicators was analyzed using the linear regression model. Bland-Altman was performed to evaluate the agreement of the two methods for measuring blood glucose. $\mathrm{P}<0.05$ was considered statistically significant.
\end{abstract}

Results: The blood glucose values measured using the arterial blood gas analyzer was significantly different from the values obtained using the rapid blood glucose meter $(\mathrm{P}=0.000)$. Regression analysis showed that $\mathrm{R} 2$ was 0.857 and $\beta$ was 0.324 ( $\mathrm{P}=0.000)$. Bland-Altman plot analysis showed that arterial blood glucose values obtained using the arterial blood gas analyzer were higher than the peripheral blood glucose values obtained using the rapid blood glucose meter on the non-infused limb, with $2.74 \%$ of dots lying outside the $95 \%$ limit of agreement and the maximum absolute value $(2.30 \mathrm{mmol} / \mathrm{L})$ of blood glucose difference within the limit of agreement. The blood glucose levels measured using the two different methods showed good agreement.

Conclusions: The difference in blood glucose values measured using the two different measurement methods was statistically significant, but the maximum absolute value $(2.30 \mathrm{mmol} / \mathrm{L})$ of blood glucose difference within the limit of agreement, which is acceptable in the clinical setting. In clinical care, it is not necessary to repeat a measure of the patient's capillary blood glucose (CBG) using the rapid blood glucose meter after the blood glucose levels have been measured with the arterial blood gas analyzer, thereby reducing the associated pain and inconvenience for the patients.

Keywords: Blood glucose; arterial blood gas analyzer; peripheral blood glucose; critically ill patient

Submitted Jan 20, 2021. Accepted for publication Mar 23, 2021.

doi: 10.21037/apm-21-354

View this article at: http://dx.doi.org/10.21037/apm-21-354 


\section{Introduction}

Blood glucose monitoring in critically ill patients is vital for avoiding the occurrence of stress-induced hyperglycemia, hypoglycemia, and excessive glycemic variability (1). In critically ill patients who are unable to express their needs effectively, low blood glucose may result in fatal hypoglycemic reactions and high blood glucose is considered to affect their prognosis. Therefore, dynamic and accurate monitoring and management of blood glucose levels is essential for the prognosis of critically ill patients.

Common blood glucose monitoring methods used in clinical practice include laboratory-based detection of glucose in venous blood, arterial blood glucose detection using a blood gas analyzer, and peripheral blood glucose detection with a glucose meter (2). Venous blood glucose measured by laboratory analysis is relatively accurate and forms the international standard for the diagnosis of diabetes. However, this method is expensive and time consuming (3). The blood glucose meters can quickly obtain the blood glucose values by measuring the peripheral vascular blood glucose, which is convenient for clinical nursing. Previous studies reported the high correlation between capillary blood glucose (CBG) and venous plasma glucose (VPG) which is suitable for bedside examination of hospitalized patients, especially in the emergency room, the operating room, and the intensive care unit (ICU) (4). Although measurement of glucose using a blood glucose meter is assumed to be straightforward, there is a $15-20 \%$ deviation due to the origin of the sample (arterial, capillary, or venous) and variations in operation methods (4). Therefore, the glucose meter is generally used for dynamic monitoring of glucose. The arterial blood gas analyzer is rarely used to determine blood glucose in the general wards due to its greater trauma and high costs. However, in ICUs, doctors usually need to adjust various ventilator parameters with the help of arterial blood gas analysis values. In addition, timely diagnosis and correction of the acid-base imbalance in critically ill patients is crucial (5).

In the ICU, real-time monitoring of blood glucose is required for critically ill patients $(6,7)$. Continuous glucose monitoring generally uses a glucometer to determine CBG levels with a schedule of Q1-Q6 h. This study compared the CBG levels measured using a glucometer and arterial blood glucose levels measured using an arterial blood gas analyzer at the same time (less than 2 minutes in between readings) of critically ill patients in China. Any differences or correlations, as well as any inconsistencies, between the
2 methods were analyzed. We present the following article in accordance with the MDAR checklist (available at http:// dx.doi.org/10.21037/apm-21-354).

\section{Methods}

\section{Study subjects}

Critically ill patients admitted to the ICU from October to December 2016 were enrolled in this study. Patients who met the following criteria were included in the study: (I) patients aged 18-70 years old; (II) during treatment in the ICU, the arterial blood gas analyzer was used and coincided with the timing of CBG determination (Q1h-Q6h); and (III) informed consent was obtained from the patient or their family. Patients who required resuscitation, patients in whom resuscitation was unsuccessful, and end-stage patients were excluded from the study. The study was conducted in accordance with the Declaration of Helsinki (as revised in 2013) and was approved by ethics board of Second Xiangya Hospital, Central South University and informed consent was taken from all the patients.

A total of 73 study subjects were enrolled, including 20 females and 53 males with a mean age of $50.29 \pm 1.39$ years. There were 26 cases who underwent thoracic surgery, 7 cases with orthopedic trauma, 10 cases with decreased saturation of pulmonary infection, 16 cases who underwent general surgery, and 14 cases who underwent dental surgery. Out of the 73 patients, 15 presented with diabetes mellitus.

\section{Blood glucose measurements}

Arterial blood glucose measurement:

Arterial blood collection operators were familiar with the procedure and received systematic training to avoid repeated puncture and mixing of venous blood. The PRE2A-PAK $2.5 \mathrm{~mL}$ arterial blood sampling kit was used for blood collection. An arterial blood collection needle was used to perform a puncture along the point with the strongest pulse at an angle of $30-40^{\circ}$. Blood was allowed to flow out naturally into the collection tube. Once $0.5 \mathrm{~mL}$ of arterial blood had been collected, the needle was withdrawn and inserted into the soft plug to prevent exposure to air which can affect the blood gas results. Pressure was applied to the puncture site for more than 10 minutes. Arterial blood gas analysis was performed using a GEM-Premier 3000 blood gas analyzer according to the manufacturer's instructions. The relevant patient information was input 
Table 1 Baseline characteristics of critically ill patients

\begin{tabular}{lc}
\hline Characteristic & Total $(\mathrm{N}=73)$ \\
\hline Age, years & $50.29 \pm 1.39$ \\
Diabetes mellitus & $15(20.55)$ \\
Male & $53(72.60)$ \\
Disease & \\
Thoracic surgery & $26(35.62)$ \\
Orthopedic trauma & $7(9.59)$ \\
Pulmonary infection & $10(13.70)$ \\
General surgery & $16(21.92)$ \\
Dental surgery & $14(19.17)$ \\
Smoking & \\
Yes & $45(61.64)$ \\
No & $28(38.36)$ \\
\hline
\end{tabular}

into the machine and the arterial blood glucose values were recorded.

Peripheral blood glucose measurement: peripheral blood samples were collected within 2 minutes before or after arterial blood sample collection. Blood glucose was measured using the Contour TS blood glucose meter and blood glucose test strips. A fingertip on the non-infusion side was selected for capillary blood collection. The fingertip was disinfected with $75 \%$ ethanol and punctured using the Sterilance Safety Blood Lancets, Press (Sterilance Medical). The fingertip was gently pinched to allow the blood to flow out naturally so that sufficient blood enters the test hole of the test strip. The peripheral blood glucose values were recorded.

\section{Statistical analysis}

Statistical analysis was performed by using SPSS 17.0 software. Measurement data were expressed as mean \pm standard deviation $(\bar{x} \pm \mathrm{s})$. Measurement data were tested for normal distribution. The correlation between indicators was analyzed using the linear regression model. BlandAltman was performed to evaluate the agreement of the two methods for measuring blood glucose. $\mathrm{P}<0.05$ was considered statistically significant.

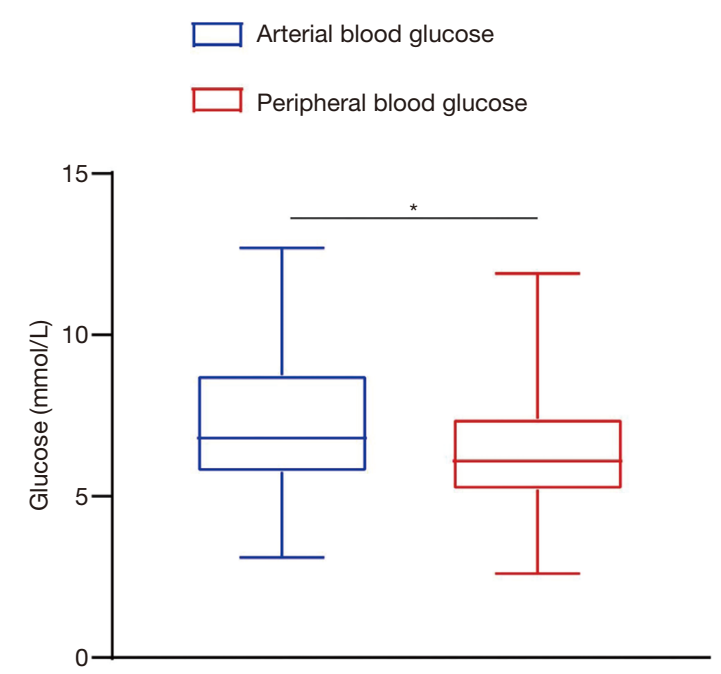

Figure 1 A comparison of arterial blood glucose and peripheral blood glucose levels in patients. * compared with arterial blood glucose group (each group was 73), $\mathrm{P}<0.05$.

\section{Results}

\section{The baseline characteristics of critically ill patients}

A total of 73 study subjects were enrolled, including 20 females and 53 males with a mean age of $50.29 \pm 1.39$ years. There were 26 cases who underwent thoracic surgery, 7 cases with orthopedic trauma, 10 cases with decreased saturation of pulmonary infection, 16 cases who underwent general surgery, and 14 cases who underwent dental surgery. Out of the 73 patients, 15 presented with diabetes mellitus (Table 1).

\section{A comparison of arterial blood glucose and peripheral blood glucose levels on the non-infusion side}

The mean arterial blood glucose was $(7.30 \pm 2.10) \mathrm{mmol} / \mathrm{L}$ and the mean peripheral blood glucose was $(6.49 \pm 1.81)$ $\mathrm{mmol} / \mathrm{L}$ for the 73 patients. There was a significant difference between the two methods $(\mathrm{P}<0.05$; Figure 1).

\section{The correlation between arterial blood glucose and peripheral blood glucose levels}

The peripheral blood glucose value (on the non-infusion side) is the dependent variable, and the arterial blood 


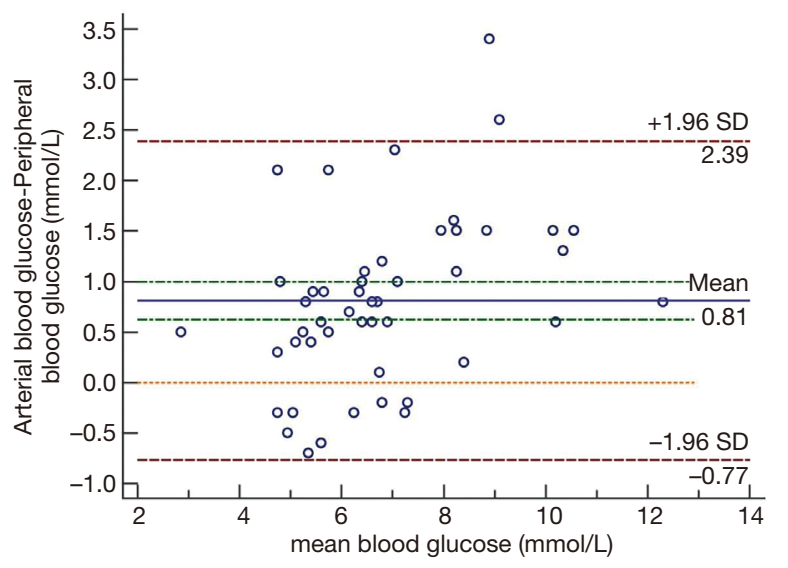

Figure 2 A Bland-Altman plot for arterial blood glucose and peripheral blood glucose. SD, standard deviation.

glucose value is the independent variable. The regression equation between arterial blood glucose values and peripheral blood glucose values (on the non-infusion side) in the 73 patients was $\mathrm{Y}=1.075 \mathrm{X}+0.324, \mathrm{~F}=425.425$ $(\mathrm{P}=0.000), \mathrm{R}^{2}=0.857, \beta=1.075, \mathrm{t}=20.626(\mathrm{P}=0.000)$. Since $85.7 \%$ of the variations in arterial blood glucose values in the samples could be explained by the peripheral blood glucose value (on the non-infusion side), this suggested that the simple linear regression model was in good agreement with the data.

\section{Agreement between arterial blood glucose and peripheral blood glucose}

Figure 2 shows the agreement between arterial blood glucose and peripheral blood glucose (on the non-infusion side). As shown in Figure 2, the upper line of agreement is $2.39 \mathrm{mmol} / \mathrm{L}$, the lower line of agreement is $-0.77 \mathrm{mmol} / \mathrm{L}$, the mean deviation is $0.81 \mathrm{mmol} / \mathrm{L}$, and the dots are randomly dispersed on both sides of the mean line with $2.74 \%$ dots outside the $95 \%$ limit of agreement. Less than $5 \%$ of the cases showed large bias between the two measurement methods, and this can be considered a small probability events. Therefore, the results indicated that the two measurement methods showed good agreement. The maximum absolute value of blood glucose difference was $2.30 \mathrm{mmol} / \mathrm{L}$ (one case with arterial glucose $8.2 \mathrm{mmol} / \mathrm{L}$ and one case with peripheral blood glucose $5.9 \mathrm{mmol} / \mathrm{L}$ ) which was within the limit of agreement and is clinically acceptable (Figure 2).

\section{Discussion}

Blood glucose measurement in critically ill patients is often affected by a variety of factors, such as instability of pathological conditions, invasive therapy, and drug administration $(5,8)$. For critically ill patients, it remains unclear which glucose measurement method is more convenient, rapid, and accurate to facilitate clinical treatments. Stress hyperglycemia is common among critically ill patients, and hence regular monitoring of blood glucose is important to maintain normal blood glucose levels and protect vital organs such as the heart and brain (9). For critically ill patients, especially those with ventilatorassisted respiration and acid-base metabolism imbalance, physicians need to adjust ventilator parameters based on the results of blood gas analysis and regulate the patient's acid-base imbalance through fluid replacement. The results in this study revealed that in critically ill patients, the difference between arterial blood glucose values and peripheral blood glucose values on the non-infusion side was $1.31 \mathrm{mmol} / \mathrm{L}$ and the difference was statistically significant, which was consistent with the results of previous studies (10). It has been reported that the blood glucose values measured by arterial blood gas analyzers are more accurate than that measured by peripheral rapid glucometers $(8,11,12)$. In this study, Bland-Altman analysis showed that the mean difference in blood glucose values measured by the two methods was not equal to 0 , suggesting that the blood glucose values measured by the arterial blood gas analyzer were different from the peripheral blood glucose values measured by Contour TS blood glucose meter. Less than $5 \%$ of dots lay outside the $95 \%$ limit of agreement and the maximum absolute value $(2.30 \mathrm{mmol} / \mathrm{L})$ of blood glucose difference was within the limit of agreement, which is acceptable in the clinical setting. The results suggested good agreement between the 2 measurement methods. For critically ill patients, after successful arterial blood extraction when performing blood gas analysis, arterial blood can be used to directly determine blood glucose with a glucometer. This then avoids the need to collect peripheral blood from patients again, thereby reducing the associated pain and inconvenience for the patients.

This study has several limitations. Patients in ICU often have complex conditions and their disease state can change rapidly. However, this study did not investigate the presence of hyperglycemia in patients with different diseases. Due to the limitation of the sample size, the effects of peripheral 
skin condition, blood glucose levels, disease severity, mean arterial pressure, and hematocrit levels on blood glucose levels were not evaluated. This may have affected the comparison between the 2 different methods of detecting blood glucose levels.

\section{Conclusions}

The difference in blood glucose values measured by the 2 different methods was statistically significant, but is acceptable in the clinical setting. Clinically, patients whose blood glucose levels have been measured by the arterial blood gas analyzer will not require a repeat CBG measurement using the rapid blood glucose meter.

\section{Acknowledgments}

Funding: None.

\section{Footnote}

Reporting Checklist: The authors have completed the MDAR checklist. Available at http://dx.doi.org/10.21037/apm-21354

Data Sharing Statement: Available at http://dx.doi. org/10.21037/apm-21-354

Conflicts of Interest: All authors have completed the ICMJE uniform disclosure form (available at http://dx.doi. org/10.21037/apm-21-354). The authors have no conflicts of interest to declare.

Ethical Statement: The authors are accountable for all aspects of the work in ensuring that questions related to the accuracy or integrity of any part of the work are appropriately investigated and resolved. The study was conducted in accordance with the Declaration of Helsinki (as revised in 2013). The study was approved by ethics board of Second Xiangya Hospital, Central South University and informed consent was taken from all the patients.

Open Access Statement: This is an Open Access article distributed in accordance with the Creative Commons
Attribution-NonCommercial-NoDerivs 4.0 International License (CC BY-NC-ND 4.0), which permits the noncommercial replication and distribution of the article with the strict proviso that no changes or edits are made and the original work is properly cited (including links to both the formal publication through the relevant DOI and the license). See: https://creativecommons.org/licenses/by-nc-nd/4.0/.

\section{References}

1. Kotzapanagiotou E, Tsotridou E, Volakli E, et al. Evaluation of continuous flash glucose monitoring in a pediatric ICU setting. J Clin Monit Comput 2020;34:843-52.

2. Hennessy AR, Reynolds RM, Walker JD. Blood glucose measurement in acute medicine: inadequate detection and management. Diabet Med 2002;19:698.

3. Huang J, Ou HY, Karnchanasorn R, et al. Clinical implication of fasting and post-challenged plasma glucose in diagnosis of diabetes mellitus. Endocrine. 2015;48:511-8.

4. Colagiuri S, Sandbaek A, Carstensen B, et al. Comparability of venous and capillary glucose measurements in blood. Diabet Med 2003;20:953-6.

5. Van Herpe T, Mesotten D. Blood glucose measurements in critically ill patients. J Diabetes Sci Technol 2012;6:22-8.

6. Holzinger U, Warszawska J, Kitzberger R, et al. Realtime continuous glucose monitoring in critically ill patients: a prospective randomized trial. Diabetes Care 2010;33:467-72.

7. Egi M, Finfer S, Bellomo R. Glycemic control in the ICU. Chest 2011;140:212-20.

8. Inoue S, Egi M, Kotani J, et al. Accuracy of blood-glucose measurements using glucose meters and arterial blood gas analyzers in critically ill adult patients: systematic review. Crit Care 2013;17:R48.

9. Magee F, Bailey M, Pilcher DV, et al. Early glycemia and mortality in critically ill septic patients: Interaction with insulin-treated diabetes. J Crit Care 2018;45:170-7.

10. Kulkarni A, Saxena M, Price G, et al. Analysis of blood glucose measurements using capillary and arterial blood samples in intensive care patients. Intensive Care Med 2005;31:142-5. 
11. Slater-MacLean L, Cembrowski G, Chin D, et al. Accuracy of glycemic measurements in the critically ill. Diabetes Technol Ther 2008;10:169-77.

Cite this article as: Deng T, Liu M, Pan L, Jiang K, Li Y. A comparison of arterial blood glucose and peripheral blood glucose levels in critically ill patients: measurements using the arterial blood gas analyzer and the rapid glucose meter. Ann Palliat Med 2021;10(3):3179-3184. doi: 10.21037/apm-21-354
12. Juneja D, Pandey R, Singh O. Comparison between arterial and capillary blood glucose monitoring in patients with shock. Eur J Intern Med 2011;22:241-4. 